\title{
Diabetic encephalopathy: beneficial effects of supplementation with fatty acids $\omega 3$ and nordihydroguaiaretic acid in a spontaneous diabetes rat model
}

\author{
Gustavo Tomás Díaz-Gerevini ${ }^{1,2}$, Alejandro Daín ${ }^{1}$, María Eugenia Pasqualini ${ }^{1}$, Cristina B. López ${ }^{1,3}$, \\ Aldo R. Eynard ${ }^{1}$ and Gastón Repossi $i^{*}$
}

\begin{abstract}
Background: Diabetic encephalopathy is a chronic complications of diabetes mellitus that affects the central nervous system. We evaluated the effect of $\omega 3$ and $\omega 6$ polyunsaturated fatty acids (PUFAs) supplementation plus the antioxidant agent nordihydroguaiaretic acid (NDGA) on the etiopathology of diabetic encephalopathy in eSS rats, a spontaneous model of type 2 diabetes.
\end{abstract}

Methods: One hundred twenty spontaneous diabetic eSS male rats and 38 non-diabetic Wistar, used as healthy control, received monthly by intraperitoneal route, $\omega 3$ or $\omega 6$ PUFA $(6.25 \mathrm{mg} / \mathrm{kg})$ alone or plus NDGA $(1.19 \mathrm{mg} / \mathrm{kg})$ for $12 \mathrm{months}$. Diabetic rats had a worse performance in behavioural Hole-Board test. Histopathological analysis confirmed lesions in diabetic rats brain tissues. We also detected low expression of synaptophysin, a protein linked to release of neurotransmitters, by immunohistochemically techniques in eSS rats brain. Biochemical and histopathological studies of brain were performed at 12th month. Biochemical analysis showed altered parameters related to metabolism. High levels of markers of oxidative stress and inflammation were detected in plasma and brain tissues. Data were analysed by ANOVA test and paired t test was used by comparison of measurements of the same parameter at different times.

Results: The data obtained in this work showed that behavioural, biochemical and morphological alterations observed in eSS rats are compatible with previously reported indices in diabetic encephalopathy and are associated with increased glucolipotoxicity, chronic low-grade inflammation and oxidative stress burden. Experimental treatments assayed modulated the values of studied parameters.

Conclusions: The treatments tested with $\omega 3$ or $\omega 3$ plus NDGA showed improvement in the values of the studied parameters in eSS diabetic rats. These observations may form the basis to help in prevent and manage the diabetic encephalopathy.

Keywords: Diabetic encephalopathy, Polyunsaturated fatty acids $\omega 3$, Nordihydroguaiaretic acid, eSS rats, diabetes mellitus

\footnotetext{
*Correspondence: grepossi@fcm.unc.edu.ar

${ }^{1}$ Biología Celular, Histología y Embriología. Facultad de Ciencias Médicas,

INICSA CONICET-Universidad Nacional de Córdoba, Córdoba, Argentina

Full list of author information is available at the end of the article
}

(c) The Author(s). 2019 Open Access This article is distributed under the terms of the Creative Commons Attribution 4.0 International License (http://creativecommons.org/licenses/by/4.0/), which permits unrestricted use, distribution, and reproduction in any medium, provided you give appropriate credit to the original author(s) and the source, provide a link to the Creative Commons license, and indicate if changes were made. The Creative Commons Public Domain Dedication waiver (http://creativecommons.org/publicdomain/zero/1.0/) applies to the data made available in this article, unless otherwise stated. 


\section{Introduction}

Diabetic encephalopathy (DE) is a chronic complication of diabetes mellitus that affects the central nervous system (CNS) and is characterized by cognitive impairment and motor dysfunctions that can cause postural balance impairment. The physiopathology of DE could be attributed to long-standing hyperglycaemia, elevated blood pressure, hyperinsulinemia, frequent and severe episodes of hypoglycaemia, and dyslipidaemia.

There is evidence linking type 2 diabetes mellitus (DM2) with low grade chronic inflammation (LGCI) [1, 2]. Hence, in a murine model of spontaneous DM2, the Stillman-Salgado (eSS) rats, we studied possible association among DE [3], neurocognitive alterations and glicolipotoxicity [4].

The concept of glucolipotoxicity refers to the combined, deleterious effects of elevated glucose, triglycerides (TG), higher energy intake and free fatty acid levels (FFA) on pancreatic beta-cell function and survival. Excessive levels of circulating FFA and glucose leads to decreased insulin secretion, impaired insulin gene expression, and in turn beta-cell death by apoptosis $[4,5]$. Several pathways have been implicated in fatty-acid inhibition of insulin gene expression, mainly by the extracellular-regulated kinase (ERK1/2) pathway, the metabolic sensor Per-Arnt-Sim kinase and the ATF6 branch of the unfolded protein response [4].

Increased lipid storage in non-adipose tissues may appear in the setting of high levels of plasma FFA or triglycerides (TG) that could lead to "lipotoxicity". Studies performed in experimental animals and humans suggested that lipotoxicity may occur due to altered energy balance as it happens in DM2, neurodegenerative diseases such as Parkinson's disease, Alzheimer's (AD), amyotrophic lateral sclerosis, and heart failure $[3,5,6]$. Accumulation of lipids in heart, skeletal muscle, pancreas and liver tissues may play an important role in the pathogenesis of these diseases [7].

Plasma concentrations of FFA are elevated in the obese subjects and in those with metabolic syndrome. These elevated FFA and non-esterified FFA levels can induce lipotoxicity, due to oxidative stress, which may impair insulin signalling and glucose response in pancreatic $\beta$-cells [4]. Experimental and clinical data suggest that saturated FFA such as palmitic acid (PA) which are present in red meat, plays a critical role in the inhibition of the insulin signalling pathway and induction of endoplasmic reticulum (ER) stress in several tissues including hypothalamic neurons. It is likely that ER stress in hypothalamic neurons may lead to AD-like pathological abnormality in primary cortical neurons. Elevated oxidative stress and FFA metabolism when it occurs in astrocytes, it may lead to an increase in their apoptotic cell death, PC12 cells and neural progenitor cells [6]. These chronic metabolic injuries on the central nervous system (CNS) in DM2, in the long run, may result in cognitive impairment and motor dysfunctions which can result in the onset of DE [3].

Epidemiological, clinical and experimental evidences revealed that mild type DM2 may result in subtle and progressive metabolic abnormalities and slow but definite onset of cognitive dysfunction especially in the presence of an imbalance between PUFAs of families $\omega 6$ and $\omega 3(\omega 6 / \omega 3)[8]$.

Based on these evidences, we evaluated the effects of intraperitoneal administration of PUFAs $\omega 6$ and $\omega 3$ alone or in combination with nordihydroguaiaretic acid (NDGA), a potent natural antioxidant compound isolated from native plant Larrea $s p$. [9], on the etiopathology of glucolipotoxicity observed in DE and focused on hippocampal tissues in a spontaneous rat DM2 model.

\section{Materials and methods}

\section{Ethical disclosure}

All animal procedures were approved by the local animal care committee (CICUAL, Facultad de Ciencias Médicas, Universidad Nacional de Córdoba, Argentina) and were developed according to international regulations.

\section{Animals}

eSS rats are a stable variety derived from Wistar strain characterized by Tarres and colleagues [10, 11]. eSS rats develop hypertriglyceridemia around 4th and DM2 at 6th months of age, which is more evident in males $[10,11]$. The study was carried out with 158 male rats: 120 diabetic eSS and 38 non-diabetic Wistar rats that formed the healthy control. Animals were kept at $22 \pm 2{ }^{\circ} \mathrm{C}$ temperature, $65 \pm 10 \%$ relative humidity, and $12 \mathrm{~h}$ light/dark cycle. Rats were provided with commercial chow pellets (GEPSA, Grupo Pilar S.A.) and water ad libitum in the animal facilities of the Instituto de Investigaciones en Ciencias de la Salud (INICSA), CONICET-UNC, Córdoba, Argentina. Efforts were made to minimize animal suffering and to reduce the numbers of animals used.

\section{Reagents}

Arachidonic Acid (AA), nordihydroguaiaretic acid (NDGA) and Xylenol Orange tetrasodium salt were purchased from Sigma-Aldrich $^{\oplus}$ (USA). Fish oil containing 35\% eicosapentaenoic acid (EPA) and 40\% docosahexaenoic acid (DHA) from Natufarma Labs (Buenos Aires, Argentina). Blood glucose and glycated haemoglobin (HbA1c) were determined by rapid test Accu-check by Roche (Switzerland). Total cholesterol (Chol), TG, C-Reactive Protein (CRP), enzymes: transaminases, pyruvic oxaloacetic, glutamic oxaloacetic and gamma glutamyltranspeptidase (GGTP) diagnostic kits were purchased from Wiener Lab $^{\bullet}$ (Argentina). Rabbit polyclonal anti-synaptophysin (SYN) antibody was purchased from 
Dako Biotechnology (Denmark). IL-6 plasma levels were measured by immuno-ELISA OptEIA ${ }^{\text {tw }}$ system and apoptosis determined by Flow Cytometry (Annexin V: PE) both detection kits were provided by BD Biosciences ${ }^{\circ}$ (USA). Fatty acids methyl ester (FAME) were identified using a commercial standard Nu-check ${ }^{\circ}$ (USA) [12]. Hippocampal IL-6 levels were determined by RT-PCR, supplies was purchased from Life Technologies (USA). Apoptotic cells in histological slides were identified by TunelS7100 ApopTag ${ }^{\circ}$ Peroxidase in situ Apoptosis Detection Kit (Germany).

\section{Experimental design}

All animals received once in a month for 12 months 0.4 $\mathrm{ml}$ of treatment by IP route. In these trials the effects of using low concentrations, but ensuring a high absorption, for a long time were studied. These concentrations also decrease the risk of adverse effects. This form of administration and doses has already been tested and published previously in murine models of metabolic syndrome and diabetes $[9,13]$. The experimental animals were subjected to the treatments detailed in Table 1.

\section{Tissue sampling}

Venous blood from fasting animals, which were slightly anesthetized with isofluorane, was taken from tail vein and centrifuged for $10 \mathrm{~min}$ at 1000x g. Plasma obtained was used for measuring various metabolic parameters: blood glucose levels, HbA1c, TGs and Cholesterol at 2, 6 and 12 months. Oxidative stress (GGTP and peroxides), LGCI markers (CRP and IL-6) and total fatty acid (FA) profile were determined in the plasma of blood obtained by cardiac puncture at the time of euthanasia.

Euthanasia was performed by overdose of isofluorane $(2,5 \mathrm{ml} / \mathrm{kg})$ and blood obtained at this time by cardiac puncture was heparinized and centrifuged for $10 \mathrm{~min}$ at

Table 1 Experimental groups and content of IP injections received as treatments

\begin{tabular}{|c|c|c|}
\hline $\begin{array}{l}\text { Rat } \\
\text { strain }\end{array}$ & $\begin{array}{l}\text { Group } \\
\text { name }\end{array}$ & Treatment $(0.4 \mathrm{ml} \mathrm{IP})$ \\
\hline eSS & Control ess & Diabetic control. Only saline isotonic solution \\
\hline eSS & $\omega 6$ & $\begin{array}{l}\text { Saline isotonic solution plus } 6.25 \mathrm{mg} / \mathrm{kg} \\
\text { of } 90 \% \text { AA }\end{array}$ \\
\hline eSS & $\omega 6+N D G A$ & $\begin{array}{l}\text { Saline isotonic solution plus } 6.25 \mathrm{mg} / \mathrm{kg} \text { of } \\
90 \% \text { AA oil }+1.19 \mathrm{mg} / \mathrm{kg} \text { NDGA }\end{array}$ \\
\hline ess & $\omega 3$ & $\begin{array}{l}\text { Saline isotonic solution plus } 6.25 \mathrm{mg} / \mathrm{kg} \text { of } \\
\text { commercial fish oil containing } 35 \% \\
\text { eicosapentaenoic acid (EPA) and } 40 \% \\
\text { docosahexaenoic acid (DHA) }\end{array}$ \\
\hline ess & $\omega 3+N D G A$ & $\begin{array}{l}\text { Saline isotonic solution plus } 6.25 \mathrm{mg} / \mathrm{kg} \text { of } \\
\text { commercial fish oil }+1.19 \mathrm{mg} / \mathrm{kg} \mathrm{NDGA}\end{array}$ \\
\hline Wistar & Wistar & $\begin{array}{l}\text { Non- diabetic control. Only saline isotonic } \\
\text { solution }\end{array}$ \\
\hline
\end{tabular}

$1.000 \times \mathrm{g}$. Brain was excised and one half was fixed in $10 \%$ formaldehyde, embedded in paraffin and $6 \mu \mathrm{m}$ slices were obtained. The other half was frozen at $-80^{\circ}$ C for further studies.

Histopathological analysis and determination of apoptosis, IL- 6 and SYN were carried out in the dissected hippocampal tissues.

The following parameters were evaluated in our study:

\section{Weight}

All the animals were examined clinically and weighted monthly under standard conditions (see summary of weight curves in Additional file 1).

\section{Behavioural testing}

Tests were carried out at the end of 6th and 12th month of age. Assays were conducted under day light between 09:00 and 11:00 a.m. after fasting overnight from 19:00 onwards. Equipment was maintained in the same position and temperature on each occasion. The Hole-Board test box consisted of a behavioral assay which provides three major patterns of behavior that broadly can be named as: exploration (in motivational terms), neophilia and neophobia (see Additional file 2). Neophilia is defined as the attraction that animals display towards a moving object or place, while neophobia is aversion that animals show towards an approaching and unknown object or place [14]. The device used in the present study was a modification from the one previously tested [15] consisting of a box $(50 \times 50 \mathrm{~cm})$, with walls and floor painted white. Four holes $(2.5 \mathrm{~cm}$ diameter) were placed in two lines in the floor, equidistant from each other and from the walls. Each rat was placed in the same corner. During 10-min length, the following behavioral criteria were recorded by the same observer: (i) moving time period in wandering; (ii) head-dip: the animal places its head into one of the holes, to a minimum depth such that the ears were at the same level of the floor of the box (an additional bout of head-dip was recorded if the animal raised its head fully out of the hole before resuming) [14-16]; (iii) rear: the animal remains stationary on its back legs and raises its fore arms off the ground, extending its body vertically in bipedestation [17].

\section{Computed Tomography (CT) imaging}

Brain CT images were obtained in four anaesthetized rats from each group, with a Picker scanner. Images were analyzed with the Fiji Image v1.51 software (USA) (see Additional file 3) [18].

Profile of total plasmatic FA by gas chromatography (GC) Plasma lipids were extracted by Folch's method and methylated with sodium methoxide. The quantification 
and identification of total FA methyl ester resultants were performed using a capillary column (BPX $20 \mathrm{~m}$ longitude, $0.25 \mathrm{~mm}$ ID, $0.25 \mu \mathrm{m}$ film, SUPELCO@, USA) in a Clarus500॰ (Perkin-Elmer ${ }^{\circ}$ ) GC and all fatty acids were identified using a commercial standard (Nu-check, $\left.\mathrm{USA}^{\circ}\right)$ [12].

\section{Histopathological analysis}

Not less than five brains were fixed in each group, processed, cut and sections stained with hematoxylin-eosin (HE) or Nissl. Hippocampal CA1 zone were evaluated by optical microscopy at $10 x$ and $40 x$ magnification in two slides by brain with 4 non-serial $6 \mu \mathrm{m}$ thick sections each ( $n=8$ sections per animal, 40 per group). To characterize the morphological changes in hippocampus, the thickness of neurons layer and cell dispersion in CA1 zone, were recorded by two examiners blinded to the condition and treatment of each rat brain. The measurements were made with the Fiji ImageJ software.

\section{Hippocampus cell isolation}

Under lens, hippocampi were removed, cut into small pieces (1-2 mm3), immersed in $0.3 \mathrm{ml}$ of $0.25 \%$ trypsin solution and incubated at $37^{\circ} \mathrm{C}$ for $30 \mathrm{~min}$. The resulting cell suspensions were washed and filtered sequentially through $40 \mu \mathrm{m}$ cell filters (BD Falcon ${ }^{\circ}$ ) and resuspended in PBS and thus, the number of cells was counted. These cells were used by apoptosis detection in flow cytometer.

\section{Apoptosis}

Apoptosis levels were determined in hippocampal isolated cells of experimental animals by Flow Cytometry using an Annexin V: PE detection kit. Apoptotic cells were also detected in histological preparations by the TUNEL technique.

\section{LGCI markers}

High-sensitive CRP was determined by immunoturbidimetry method with latex particles. Plasmatic IL-6 levels were detected by immunoassay and quantified by means of calibration curve using a commercial standard.

In hippocampus cells IL-6 mRNA expression was assayed by PCR-RT. Total RNA was extracted with TRIzol - (Life Technologies) and was quantified using a fluorometric method (The Qubit ${ }^{\circledR}$ 2.0 Fluorometer, Life Technologies). Samples of DNA copy was amplified in triplicate with a StepOnePlus RT-PCR System (Applied Biosystems). For each sample, the amount of mRNA was normalized against the bulk of mRNA of $\beta$-actin (used as reference gene). Oligonucleotide sequence was:

IL6_FwTAGTCCTTCCTACCCCAACTTCC,

IL6_RevTTGGTCCTTAGCCACTCCTTC;

$\beta$-actin_FwCCGCATCCTCTTCCTCCCT,

\section{$\beta$-actin_RevGCCACAGGATTCCATACCCAG [19].}

\section{Oxidative stress biomarkers}

GGTP specific activity was determined spectrophotometrically at $405 \mathrm{~nm}$ in plasma and brain tissue by a commercial kit (Wiener Labs, Argentina). Peroxides levels were measured in plasma by xylenol orange method [20].

\section{Hepatic function}

As a way to determine gross perturbations in the physiology of liver, plasmatic levels of hepatic enzymes were evaluated.

\section{Statistical analysis}

The data obtained from experiments were analyzed by ANOVA test for the comparison of means. Measurements of the same parameter at different experimental times were analyzed by paired $t$ test. A value of less than $0.05(p<0.05)$ was used to define significant differences in all tests. Statistical tests were performed using the InfoStat 3.1 software (Grupo InfoStat, FCA, Universidad Nacional de Córdoba, Argentina).

\section{Results \\ Weight}

The eSS rats showed a greater increase in their body weight in the first month compared to Wistar group, but all groups reached a similar weight by the end of the study period. As expected in this model no obesity was observed (see Additional file 1).

\section{Glycaemia and Oral glucose tolerance test (OGTT)}

All eSS rats developed diabetes within 6th month of life (120/120: 100\%). There were no differences in the plasma glucose levels among diabetic eSS groups. Wistar group maintained normal values (Fig. 1a and b).

\section{HBA1c}

In all eSS rats reaching the 6th month of age, HbA1c levels were abnormal i.e. $>6 \mathrm{mg} / \mathrm{dl}$, without any significant difference among all treatments studied. On the other hand, HbA1C remained normal $(\leq 6 \mathrm{mg} / \mathrm{dl})$ in Wistar rats (Fig. 1c).

\section{Triglyceridemia}

In groups of eSS rats, TG showed normal values at 2 months of age. But by 6th month of age TGs were abnormal that remained high even at 12th month of age. Even though $\omega 3+$ NDGA treatment induced a decrease in TG values, these values were not statistically different from the Control eSS. TG in Wistar group continued within normal range (Fig. 2a). 


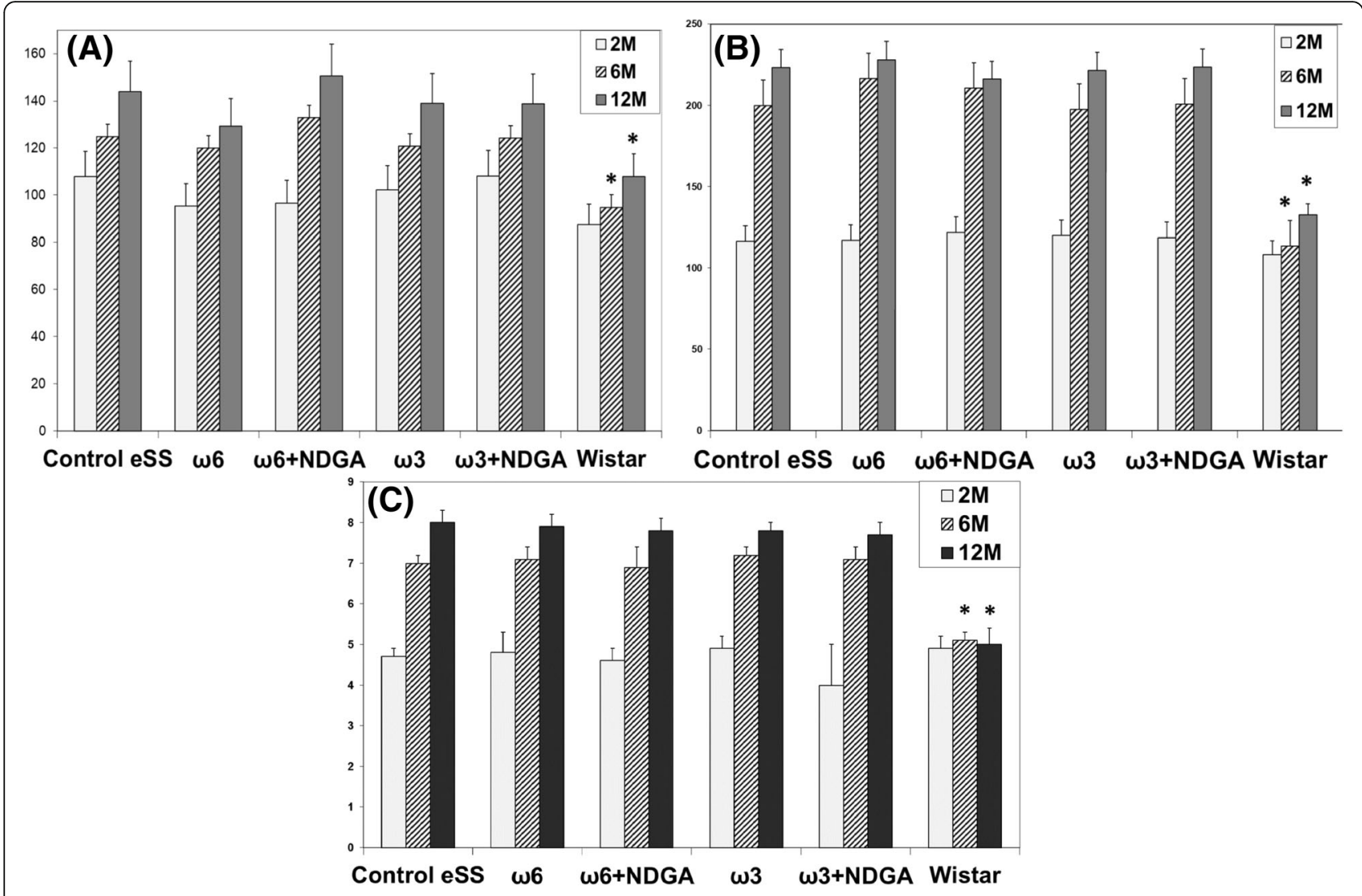

Fig. 1 Glycaemic metabolism. OGGT of experimental animals performed at 2, 6 and 12 months. a Fasted glycaemia (mg/dl), b postprandial glycaemia (mg/dl) and $\mathbf{c ~ H b A 1 c ~ l e v e l s ~ ( \% ) . ~ D a t a ~ a r e ~ e x p r e s s e d ~ a s ~ m e a n ~} \pm$ SEM. * Indicate significant difference to Control eSS, $p \leq 0.05$

\section{Cholesterolemia}

Cholesterol values persisted within the normal range in all groups (Fig. 2b).

\section{Plasmatic profiles of total fatty acids}

Total FA profiles in plasma at 12th month of age showed variations among treatment groups. As shows the Table 2, results grouped in a summarized form according to their degree of unsaturation, showed significant differences that could be related to the type of PUFAs administered. It is evident from these results that administration of $\omega 6$ and $\omega 3$ PUFAs produced an increase in their respective total PUFAs, in the groups that received them. NDGA addition produced an increase in PUFAs levels (Table 2).

\section{Cognitive-behavioural data: Hole-board test}

At the end of the 6th month of the study, eSS rats were significantly more active in the behavioural parameters compared to non-diabetic rats (Fig. 3). When the accomplishments of tasks were compared, total records of locomotion, frequency of rearing, and head-dip at the end of 12 th month, they were significantly worse in untreated diabetics rats. These parameters showed improvement at the end of 12th month in those which were administered $\omega 3$ alone or $\omega 3+$ NDGA. On the contrary, rats treated with $\omega 6$ alone showed a diminution in their performance in the Hole-Board test (Fig. 3). Comparative values recorded by healthy Wistar rats between 6th and 12th month of age remained normal. On the other hand, eSS rats showed a decline in their performance over time. At the end of 12th month, Control eSS untreated rats showed poorest performance.

\section{Histopathological analysis}

eSS control animals did not show amyloid deposits in brain tissues or vascular lesions linked to atheros clerosis. Additionally, densitometric analysis of CT images showed areas of less homogeneity in eSS diabetic rat brains compared to Wistar rats. These alterations were less noticeable in animals that received $\omega 6+$ NDGA or $\omega 3+$ NDGA or even only $\omega 3$ (see Additional file 3).

Diabetic Control eSS, $\omega 6$ and $\omega 6+$ NDGA rats showed white areas in brain tissue images, which may be 

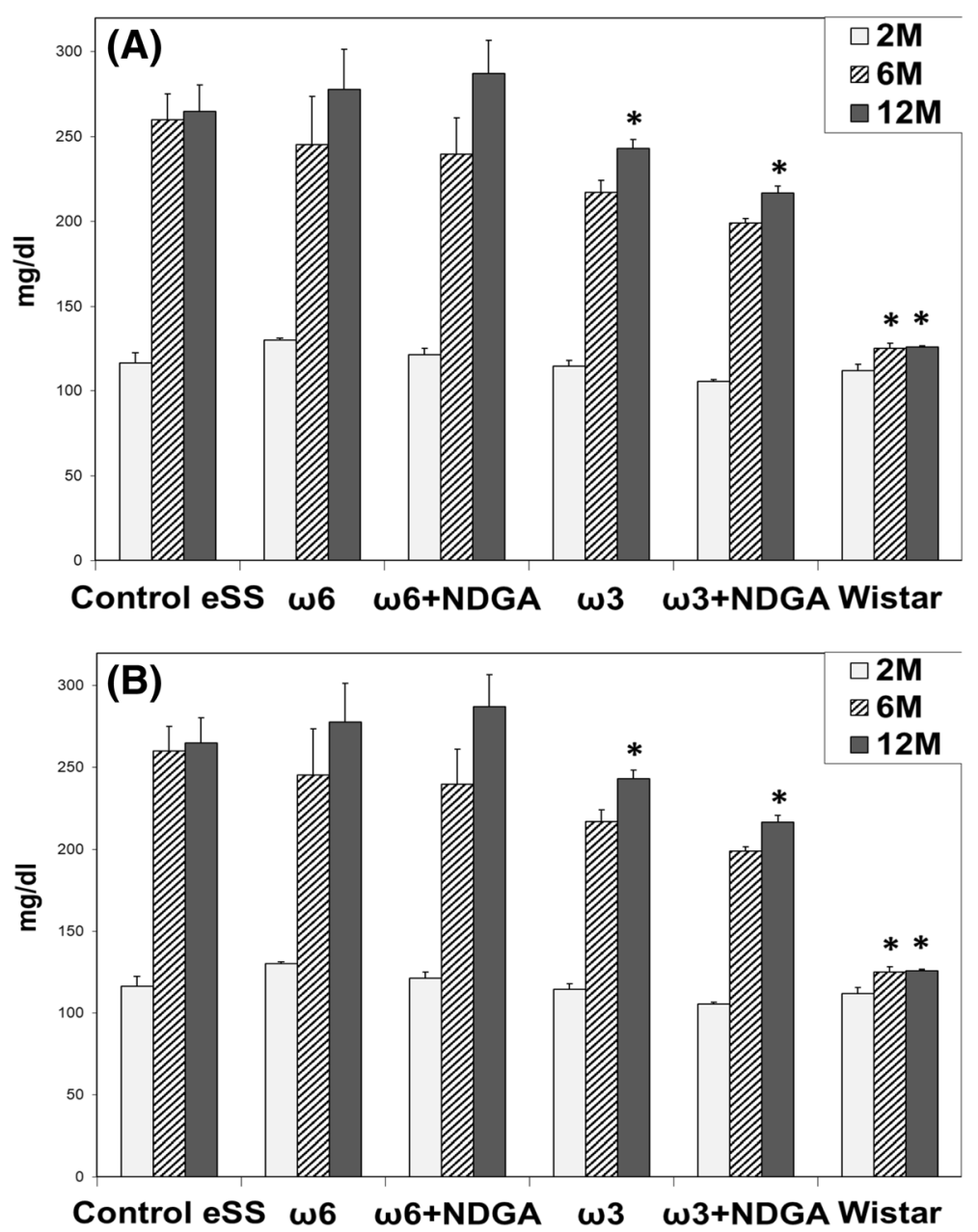

Fig. 2 Blood lipids. Levels of plasmatic (a) triglycerides and $\mathbf{b}$ total cholesterol of animals at 2, 6 and 12 months. Data are expressed as mean \pm SEM. * Indicate significant difference to Control eSS, $p \leq 0.05$

consistent with scattered spongiosis and gliosis, as well as greater thickness and cell dispersion of the hippocampal CA1 zone could be visualized (Fig. 4). The burden of these alterations was lower in eSS rats treated with $\omega 3$ alone or plus NDGA. These morphological abnormalities were not observed in healthy control Wistar rats.

\section{Apoptosis}

Flow cytometry and TUNEL in dissected hippocampus of $\omega 6$ and $\omega 6+$ NDGA and eSS control rats showed significantly higher number of apoptosis than $\omega 3$ alone or $\omega 3+$ NDGA groups. Animals treated with $\omega 3$ PUFAs had the lowest number of hippocampal neuronal apoptosis (Fig. 5).

Table 2 Plasma total fatty acid profiles by GC

\begin{tabular}{|c|c|c|c|c|c|c|c|c|c|c|c|c|}
\hline \multicolumn{13}{|c|}{ Experimental groups } \\
\hline & \multicolumn{2}{|c|}{ Control eSS } & \multicolumn{2}{|l|}{$\omega 6$} & \multicolumn{2}{|c|}{$\omega 6+N D G A$} & \multicolumn{2}{|l|}{$\omega 3$} & \multicolumn{2}{|c|}{$\omega 3+N D G A$} & \multicolumn{2}{|l|}{ Wistar } \\
\hline \multicolumn{13}{|l|}{ Fatty acids } \\
\hline SFA & $23,56^{\#}$ & $\pm 2,28$ & $45,89^{\#^{*}}$ & $\pm 3,46$ & $38,20^{\#^{*}}$ & $\pm 3,18$ & $22,20^{\# *}$ & $\pm 0,34$ & $25,00^{\#^{*}}$ & $\pm 2,90$ & $27,90^{*}$ & $\pm 2,53$ \\
\hline MUFAs & $27,60^{\#}$ & $\pm 2,60$ & $17,46^{\#^{*}}$ & $\pm 1,51$ & $24,50^{\#^{*}}$ & $\pm 1,29$ & $33,30^{*}$ & $\pm 0,25$ & $45,40^{\#}$ & $\pm 0,76$ & $22,50^{*}$ & $\pm 1,36$ \\
\hline PUFAs w6 & 38,20 & $\pm 3,65$ & $30,37^{\#^{*}}$ & $\pm 0,10$ & $23,70^{\#^{*}}$ & $\pm 1,09$ & $29,00^{*}$ & $\pm 0,04$ & $12,00^{*}$ & $\pm 0,44$ & $38,00^{*}$ & $\pm 3,18$ \\
\hline PUFAs w3 & $4,00^{\#}$ & $\pm 0,54$ & 1,83 & $\pm 0,01$ & 6,20 & $\pm 0,33$ & 15,10 & $\pm 0,16$ & $19,30^{\#}$ & $\pm 0,48$ & $16,40^{*}$ & $\pm 0,36$ \\
\hline PUFAs Total & 42,20 & $\pm 2,09$ & $32,20^{\#}$ & $\pm 0,05$ & $29,10^{\#}$ & $\pm 0,71$ & 44,00 & $\pm 0,10$ & $31,10^{\#}$ & $\pm 0,46$ & $54,30^{*}$ & $\pm 1,77$ \\
\hline Ratio $\omega 6 / \omega 3$ & $9,55^{\#}$ & & $16,60^{\#}$ & & $3,82^{\#^{*}}$ & & $1,92^{*}$ & & $0,62^{*}$ & & $2,32^{*}$ & \\
\hline
\end{tabular}

Profiles was determined at $12 \mathrm{TH}$ month of age in experimental groups

\#Indicate significant difference to Wistar, $p \leq 0.05$. " Indicate significant difference to Control eSS, $p \leq 0.05$ 


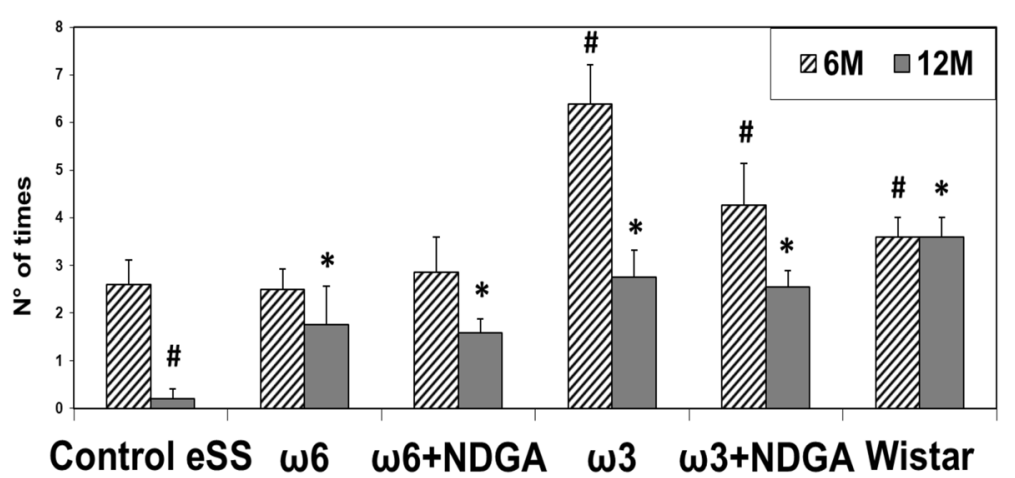

(A)

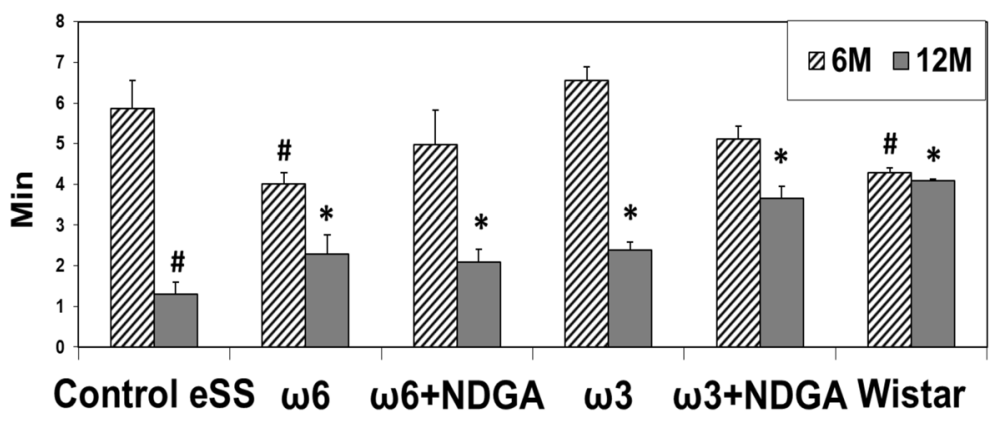

(B)

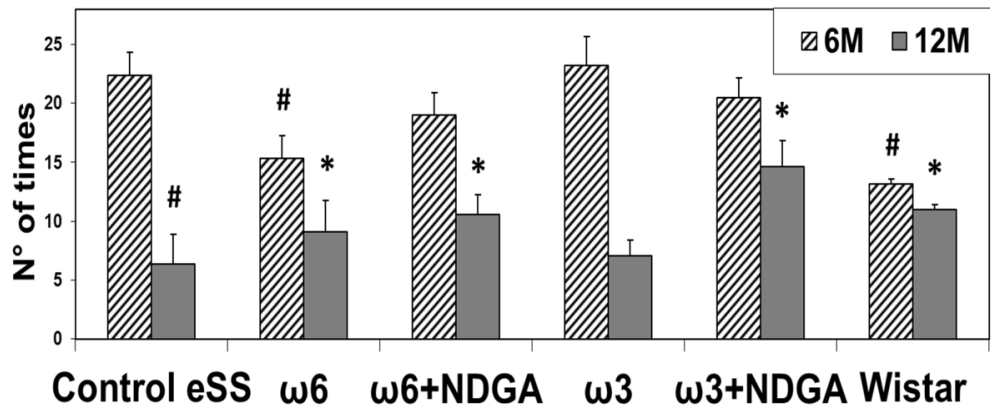

(C)

Fig. 3 Behaviour Evaluation by Hole Board Test. a Head deeping, b Time Movement and c Rearing. Data are expressed as mean \pm SEM. \# Indicate significant difference to Control eSS at 6th month, $p \leq 0.05$. ${ }^{*}$ Indicate significant difference compared to Control eSS at 12 th month, $p \leq 0.05$

\section{Synaptophysin analysis}

Control eSS, $\omega 6$ alone or plus NDGA groups showed low immunostaining for SYN in hippocampus. Rats receiving $\omega 3$ or $\omega 3+$ NDGA showed levels of labelling like those observed for healthy Wistar rats (Fig. 6).

\section{Inflammation parameters}

Plasma CRP levels were significantly higher $(p<0.05)$ in untreated eSS rats compared to healthy Wistar rats. Both groups of animals that received NDGA and those rats were treated with $\omega-3$ alone, showed lower levels of CRP (Fig. 7). IL-6 values were significantly increased in plasma and hippocampus in Control eSS group. On the other hand, Wistar rats had the lowest levels of IL-6 values. Those animals that received treatment only with $\omega 6$ or $\omega 3$ showed an insignificant reduction, which became significantly decreased when NDGA was also administered (Fig. 7). In plasma, groups that were treated only with PUFAs or PUFAs plus NDGA showed a diminution of IL-6 levels and this was most evident in those that received $\omega 3$ PUFAs.

\section{Oxidative stress}

Gamma-glutamyltranspeptidase activity

Plasma and brain tissues of eSS rats showed higher levels of GGTP activity, a biomarker of oxidative stress. 

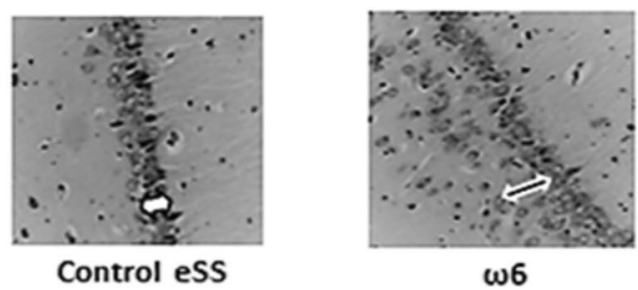

$\omega 6$

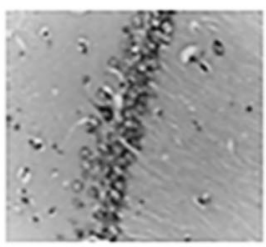

w3

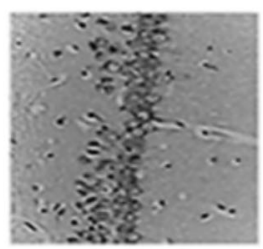

$\omega 3+N D G A$

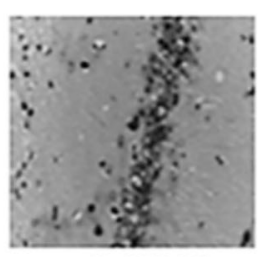

$\omega 6+N D G A$

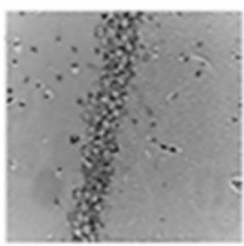

Wistar

(A)

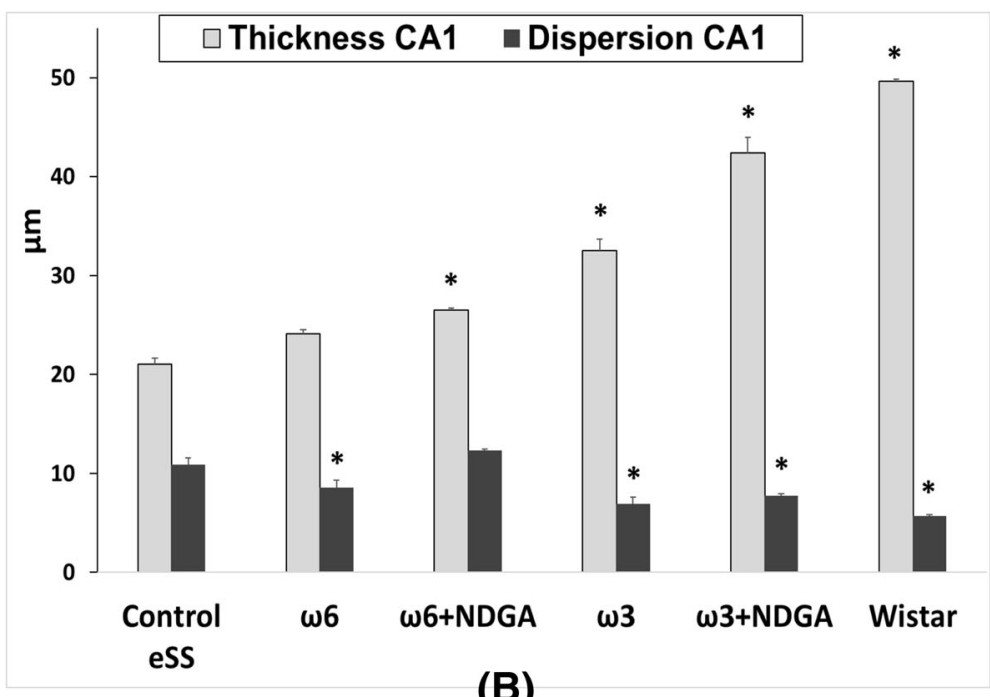

(B)

Fig. 4 Histological sections of hippocampus. a Cell dispersion (black arrow) and thickness (white arrow) of the hippocampal CA1 zone, at 12 months of aging. Nissl 40x. b Histopathological analysis. Data are expressed as mean \pm SEM * Indicate significant difference to Control eSS at the end of 12 months, $p \leq 0.05$

Rats that received NDGA or $\omega 3$ alone showed a significant decrease of plasma GGTP activity. $\omega 6$ alone treatment did not produce any significant effect on GGTP activity in the brain (Fig. 8a).

\section{Peroxides}

Higher levels of plasmatic peroxides (hydro- and lipo-) were recorded in control diabetic eSS and $\omega 6$ treated groups compared to healthy Wistar rats. Treatment with $\omega 3+$ NDGA decreased hydro- and lipo-peroxides levels in eSS rats. $\omega 6+$ NDGA treatment diminished plasmatic lipo-peroxides, whereas $\omega 3$ alone decreased only levels of hydroperoxides (Fig. 8b).

\section{Hepatic enzymes}

To determine gross perturbations in the morpho-physiology of liver, plasmatic pyruvic oxaloacetic and glutamic oxaloacetic enzymes were assayed. Their levels were within normal values. Liver histopathological study showed neither steatosis nor any other hepatic lesion (results not shown). These data indicate that treatments employed in the present study have no effect on hepatic structure and function.

\section{Discussion}

Behavioural performances, metabolic perturbations, images abnormalities and histopathological changes recorded in present work revealed that extensive brain damage compatible with DE is likely to occur in DM2 $[4,21,22]$.

Profiles of plasmatic FA were modified in the experimental animals by treatments employed. The ratio of plasmatic $\omega 6 / \omega 3$ was different among groups as shown in Table 2. It was higher in diabetic compared to Wistar animals. Interestingly $\omega 6$ group showed values no different 

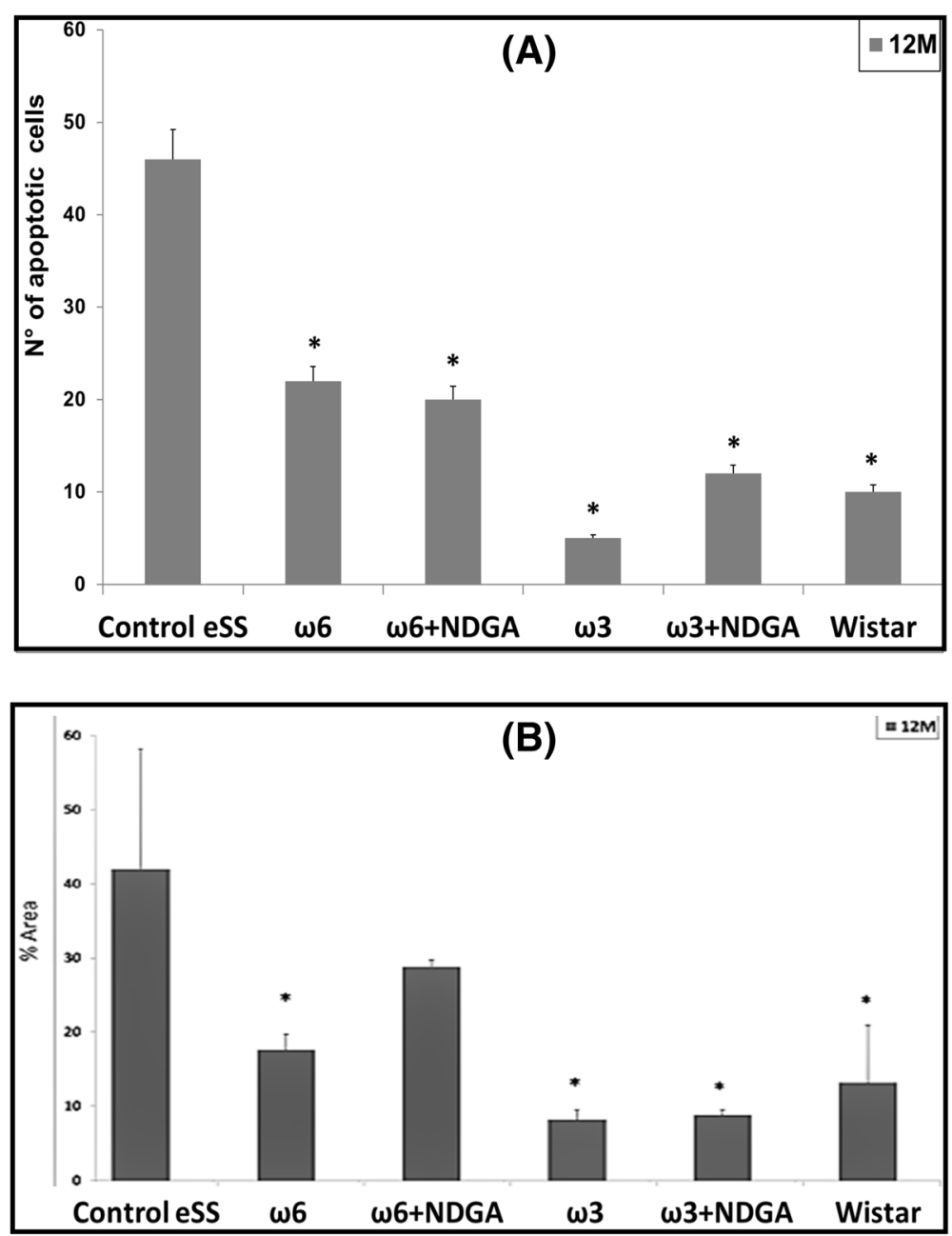

Fig. 5 Apoptosis. Hippocampal apoptosis in animals at the end of 12 months (a) by flow cytometry, $\mathbf{b}$ by TUNEL. Data are expressed as mean \pm SEM. * Indicate significant difference to Control eSS, $p \leq 0.05$

from Control diabetic eSS rats. On the other hand, those animals administered with $\omega 3$ exhibited lower values of this ratio, similar to non-diabetic Wistar rats, which showed further decrease when NDGA was added. These findings suggest that low doses of fish oil when given for a long period of time produce desirable effects on PUFAs homeostasis as previously proposed $[3,23]$.

Activity of desaturases that are needed for fatty acid synthesis are known to be affected in DM2 animals and humans [24] that could explain differences in the levels of various PUFAs noted in the plasma among control, diabetic eSS and Wistar groups and improvements induced by low doses of $\omega 3$ PUFAs administration alone or in combination with NDGA.

DM2 is a chronic plurimetabolic disease whose mechanism of injury are associated with glucolipotoxicity [1, 4-7] an abnormal condition that is associated with increased activity of GGTP, hypertriglyceridemia, hyperglycaemia, insulin resistance and elevated levels of saturated free FA. All these metabolic abnormalities can, in turn, enhance apoptosis of pancreatic $\beta$-cells and neurons in the brain [4, 23-26]. PUFAs, especially $\omega 3$ have generally been considered to have a protective function in view of their anti-inflammatory and anti-apoptotic action $[3,4]$, as confirmed in the present study [3, 26-29]. Tarres and co-workers $[10,11]$ reported that eSS rats are a suitable model to study DM2 induced metabolic damage in view of the fact that these animals showed early development of hypertriglyceridemia and subsequently hyperglycaemia similar to what is observed in patients with DM2. eSS rats are not hypertensive and also did not show obesity or atheroma that could be related to neuronal damage $[1,3]$. This implies that neuronal changes seen in this eSS diabetic model are solely due to metabolic changes induced by DM2.

Our results suggested that $\omega 3$ PUFAs ameliorate biochemical abnormalities associated with DM2 pathology [8]. The recorded plasma increment of MUFA in eSS rats compared 


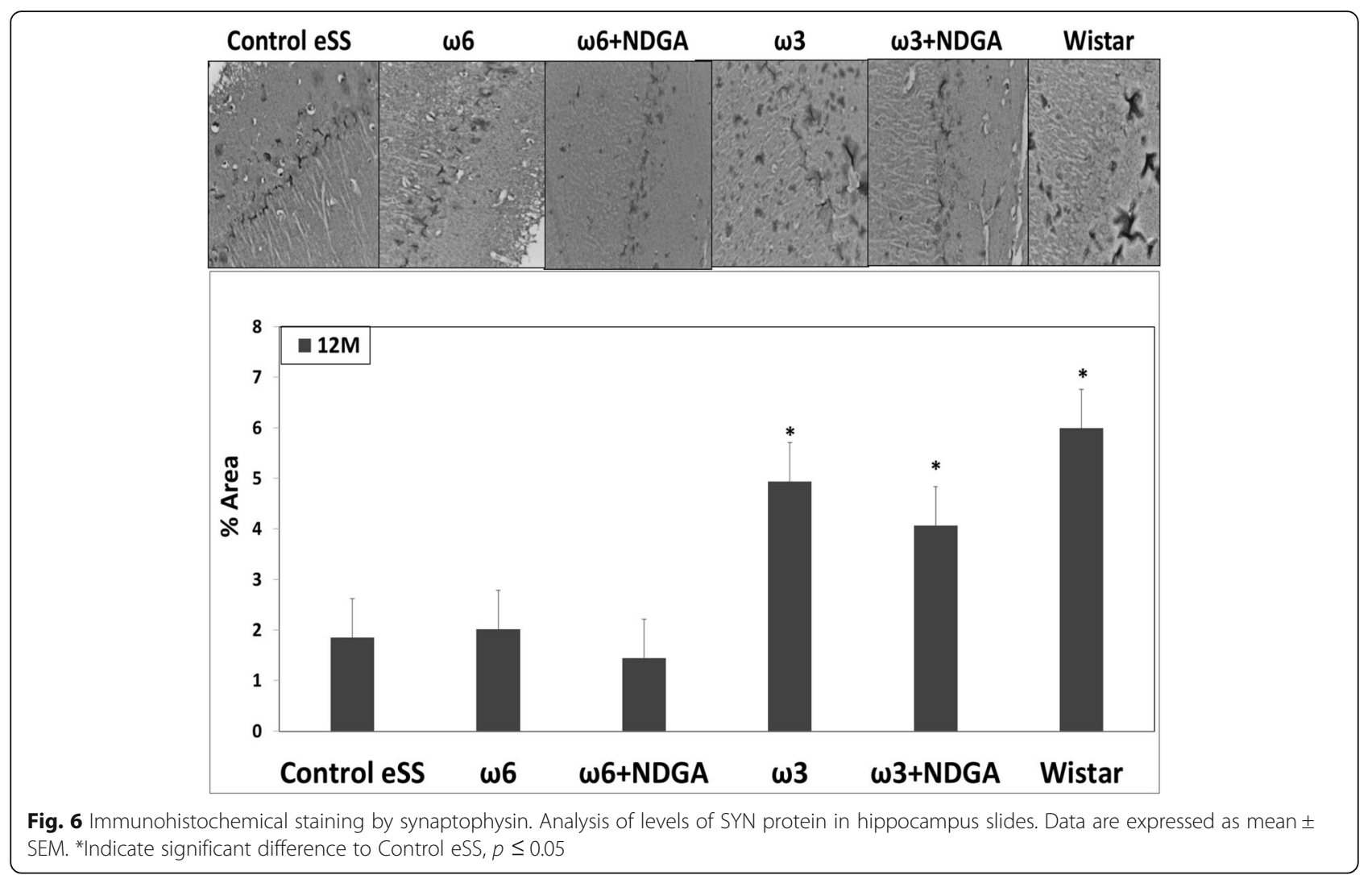

to normal Wistar rats may be seen as a result of an attempt to elongate and desaturate precursor $(18: 1 \omega 9, \mathrm{OA})$ to long chained highly unsaturated FA, as previously discussed elsewhere [30,31]. Out data reinforce the importance of certain dietary fats as potential risk factors associated with DM2 and its associated plurimetabolic nature.

At weaning, eSS were metabolically normal and even heavier than their normal Wistar counterpart (see Additional file 1). Only after weaning, they showed a progressive increase in glycaemia, OGTT and $\mathrm{HbA1c}$ values up to 6 months of age, values which remained elevated till the end of the study (Fig. 1). Since, by the end of 6th months of age eSS rats show an increase in TG, but not in Chol (Fig. 2), a phenomenon that could be related to glucolipotoxicity $[3,4,25,26]$ as previously observed by Tarres group [10, 11]. However, addition of $\omega 3$, $\omega 6$ PUFAs and NDGA treatments did not modify significantly these parameters.

Nevertheless, groups treated with $\omega 3$ alone or $\omega 3+$ NDGA showed improvements in the behavioural test, tomographic images, LGCI, oxidative stress and cell damage. These results could be related to a decrease in TG, CRP, IL-6, oxidative stress and apoptosis burden. Indeed, when cognitive behaviour was analysed by Hole-Board Test, eSS diabetics rats were more active than non-diabetic rats during the first weeks of age
(Fig. 3), a finding previously described in diabetic mice by Toth et al. [20]. Hypermotility and hyperphagia may be linked to hunger for glucose thus triggering increased exploratory wandering. On the other hand, at the end of the experimental period decreased activity in all these three parameters: "Head dipping", "Time Movement" and "Rearing" were different among the groups studied and Control eSS rats showed the most abnormalities (Fig. 3). Integrity of hippocampal nuclei is crucial for accurate control of rearing in challenging novel environments $[15,18]$. Head dipping recruits and processes new spatial information [16], exploratory behaviour and neophiliac [14]. Normal moving is associated with a motor efficient system, especially with motivational-behavioural stimuli and others as functional plasticity processes which have origins in the dentate gyrus [17]. Our work showed that untreated eSS animals showed worse results in the elapsed experiment period, while the performance of animals treated with $\omega 3+$ NDGA recovered appropriate performance with marks similar to those obtained in healthy Wistar rats (Fig. 3). Hippocampus manages sensory afferents and synaptic mechanisms underlying certain types of fast learning as well as establishing crossed links to motivational, emotional, executive, and sensori-motor functions [32]. Experienced geriatricians reported that those suffering with long-standing hyperglycaemia in the form of DM2 show subtle changes in 

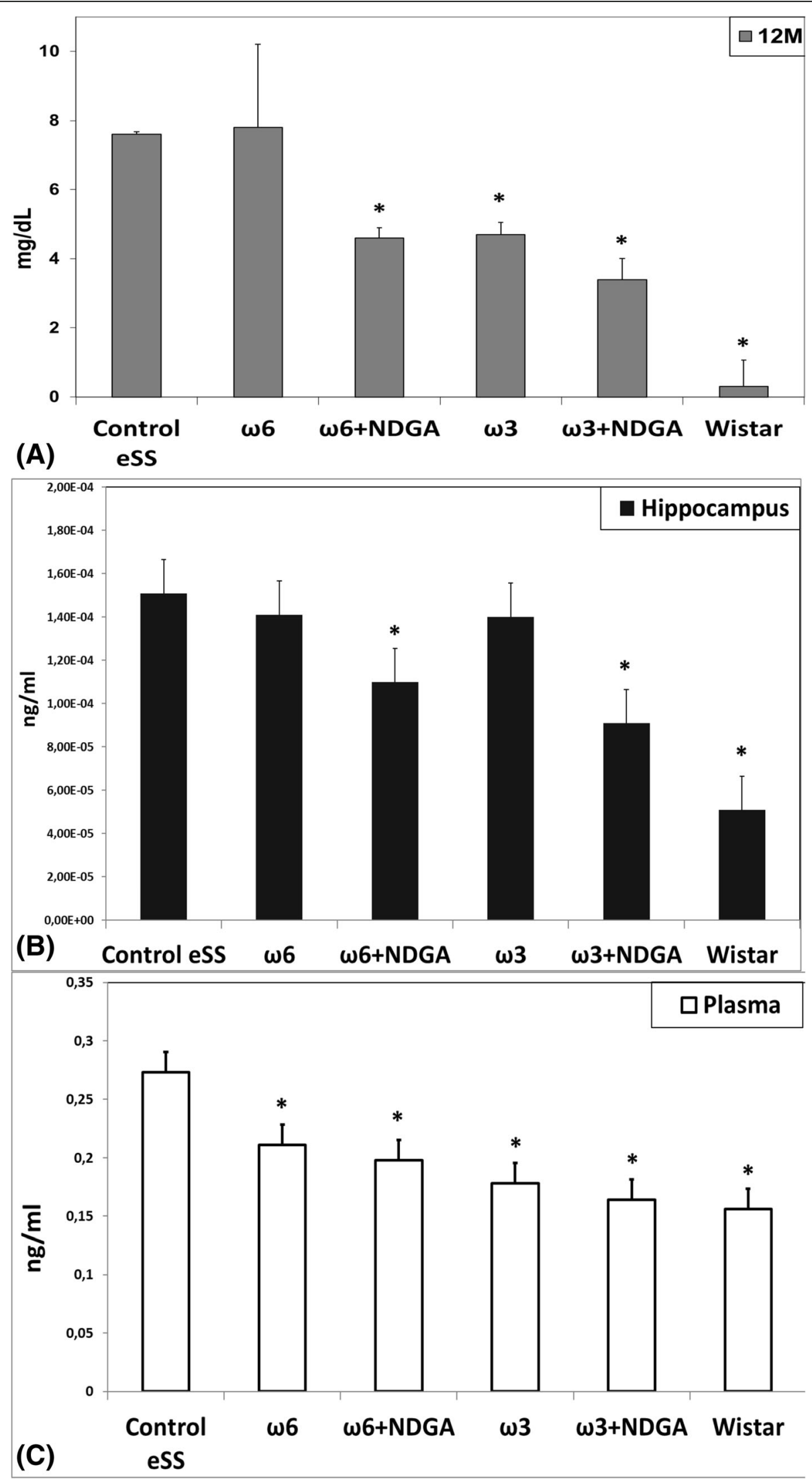
Fig. 7 Levels of inflammatory markers. a Plasmatic high-sensitive CRP concentration. Levels of IL-6 measured in b plasma and $\mathbf{c}$ hippocampus. Markers were measured in animals at 12 month. Data are expressed as mean \pm SEM. * Indicate significant difference to Control eSS, $p \leq 0.05$

their usual behaviour. Many long standing DM2 patients exhibit difficulties in learning and progressive apathy, a pleomorphic scenario that becomes difficult to differentiate from depression or other neurodegenerative diseases [33]. Motor perturbations in patients are like those observed in eSS rats that are in agreement with data reported by Zhao et al. [34]. These researchers observed deficit of learning and memory in diabetic rats which they related to increased oxidative stress, endoplasmic reticulum stress and CNS cell apoptosis [34]. Whays et al., also observed altered behaviour in diabetic rats, concluding that a "depression-like" conditions and oxidative stress developed in chronic diabetic animals [35].
CT brain images showed in Control eSS rats and those treated with $\omega 6$ scattered patterns of heterogeneity (see Additional file 3) which resemble to those observed in long standing diabetics patients suffering from white matter lesions [20-22].

Thus, LGCI condition plays a key role as we proposed previously [3], since NDGA is an anti-inflammatory compound. Neuroimaging obtained in present work gives plausible anatomical comparative evidences similar to those observed in elderly DM2 patients, namely lacunar infarcts and cortical atrophy, giving further explanation for the cognitive declining seen in long standing diabetics patients [20-22].

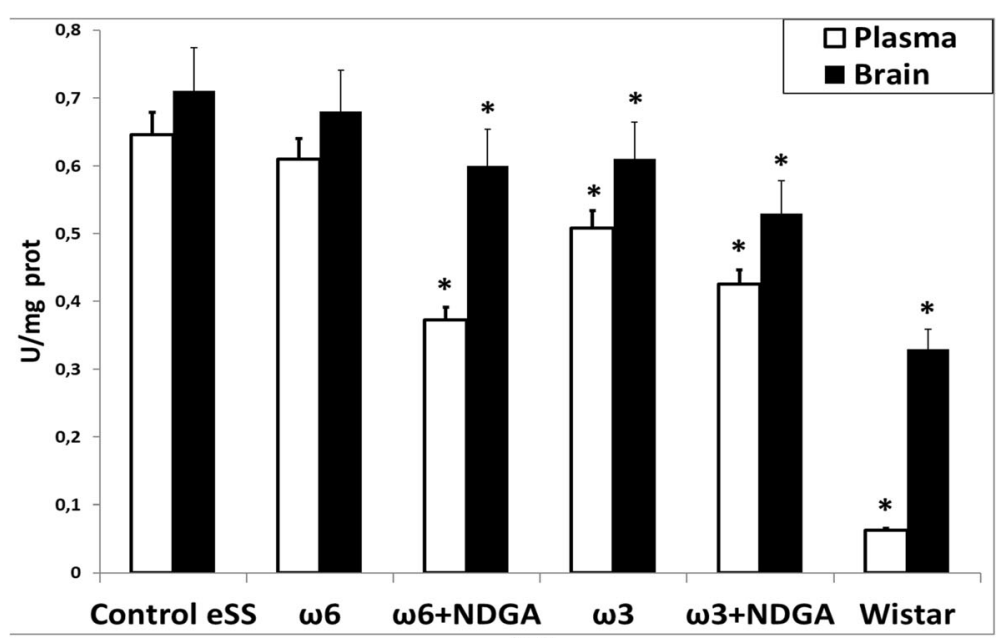

(A)

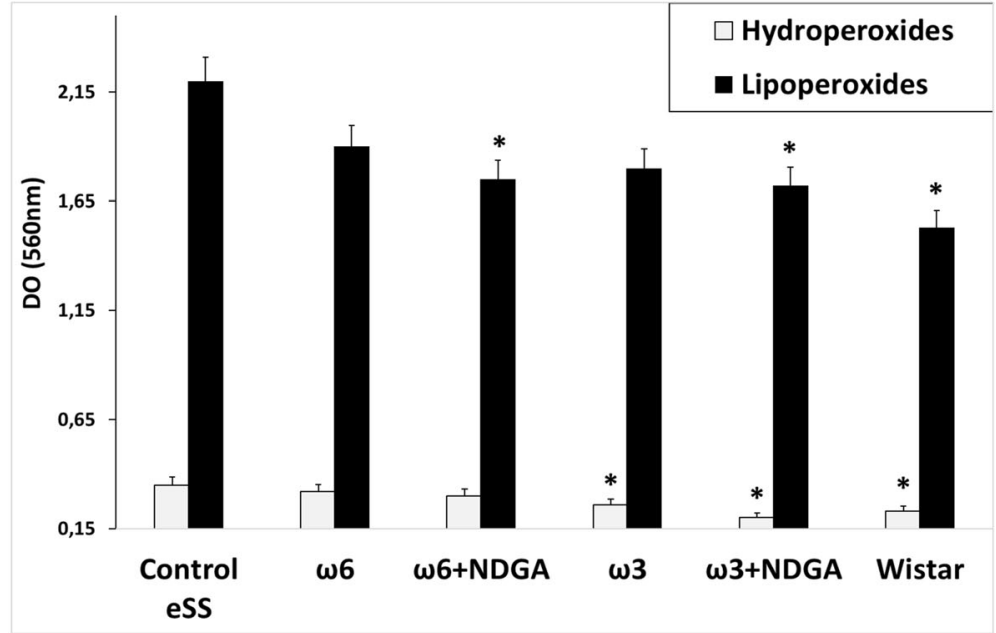

(B)

Fig. 8 Stress oxidative markers. Markers were determined in animals at 12 month. a GGTP enzyme activity measured in plasma and brain tissue. b Plasmatic hydroperoxides and lipoperoxides assays. Data are expressed as mean \pm SEM. * Indicate significant difference to Control eSS, $p \leq 0.05$ 
Histopathological abnormalities recorded in our work support described white matter and hippocampal lesions. Structural dispersion of neuronal layers, spongiosis, gliosis, cytoplasmic vacuolization, apoptosis and perikarion vacuolization- a known but not understood phenomenon described in DE were observed at hippocampus specially on the dentate gyrus and CA1 zones in eSS rats (Figs 4 and 5). These findings were previously described in type-1 and 2 diabetic rats by Amin [36]. Perhaps, it could be associated with the Golgi-ER axis stress, with their consequent "swelling" by increased synthesis and storage of abnormal glycoproteins as proposed by Zhao et al. [34]. The presence of abnormal cellular dispersion led us to consider damage to synapses associated with ED, as is seen in Alzheimer's disease. Observed pyramidal layer CA1 zone thickness may be related to increased apoptosis. It is well known that synapse damage is a key progressive lesion in dementia [37]. SYN, a crucial presynaptic protein, also decreased in aged diabetic rats compared with age-matched controls [38]. SYN and synapses are affected in imbalanced neuronal metabolism in aged diabetic rats [39]. Coincidently immunostaining of SYN was less evident in our Control eSS as well as in animals treated with $\omega 6$, whereas animals administered with $\omega 3$ alone or plus NDGA, showed average labelling similar to Wistar rats (Fig. 6). So, synapse preservation maybe the results of the beneficial effects of the combination of $\omega 3$ and NDGA treatment in this model. Besides, CRP and IL-6 were elevated in brain and plasma in eSS groups (Fig. 7) giving support to observed structural changes and LGCI state. Taking all these evidences into consideration, it is supported that a pathophysiological association exists between LGCI and progressive brain damage seen in elderly DM2 patients [3, 40, 41]. Another pathophysiological feature that is verified in the present study which sustain the above conclusion was the elevated GGTP values recorded in plasma and brain of eSS rats (Fig. 8a). On the other hand, values of hepatic enzymes were normal suggesting that there is no hepatic damage that could account for high lipo- and hydro-peroxides values determined in our eSS rat model (Fig. 8b). These assays are reliable markers of cellular oxidative stress. Indeed, these are important and frequent mechanisms by which complications due to type 2 DM might occur [42], even in prediabetes state [43] as a result of metabolic dysfunction [44] that can cause DNA damage and neuronal apoptosis [45, 46]. Based on the results of the present study, it is tempting to suggest that exogenous supply of $\omega 3$ PUFAs may be a promising protective measure to prevent oxidative damage to CNS in elderly DM2 subjects [46-48].

DE pathophysiology is related to longstanding insulin resistance, glucolipotoxicity, LGCI and oxidative stress, which can trigger activation of AKT enzyme pathway [49]. The observed improvement in eSS rats by the addition of $\omega 3$ PUFAs may result in better insulinsensitive mechanism either by an increase of IP3, the natural AKT activator and/or the ability of $\omega 3$ PUFAs, mainly DHA, to bind to nuclear peroxisome proliferator activated receptors (PPAR $\alpha$ and PPAR $\gamma$ ) that, in turn, improves learning and memory [49].

There is increasing evidence that pro-inflammatory cytokine-induced response, linked to LGCI state, is closely involved in the pathogenesis of DM2. Elevated circulating inflammatory markers such as CRP and IL-6 may precede the development of DM2 and associated complications $[50,51]$. In our eSS rats, LGCI significantly increased with age. In addition, increased levels of IL- 6 in plasma and hippocampus tissues was also noted (Fig. 7b and c). Interestingly, in eSS rats treated with $\omega 3$ PUFAs and NDGA showed a decrease in these indices $[52,53]$.

In summary we observed that in eSS diabetic rats a strong link exists among increased oxidative stress and LGCI together with histopathological abnormalities, and apoptosis that could be related to the clinical complications observed in DE in elderly DM2 subjects. Our results also suggest that administration of low doses of $\omega 3$ PUFAs and NDGA can protect against neuronal damage that occurs due to long standing DM2.

\section{Conclusions}

Neurocognitive, behavioral, biochemical and morphological perturbations observed in this model of DM2 (eSS rats) are compatible with changes seen in DE. Our results support that pathophysiology of DE, which is associated with glucolipotoxicity, chronic inflammation and high oxidative stress markers, can be neutralized by $\omega 3$ plus NDGA administration leading to significant improvements from features of DE.

\section{Additional files}

Additional file 1: Hole-Board test box. Illustration of the three

Additional file 2: Table of GLC data. Complete profiles of plasma total fatty acids of 12-month-old rats. (TIF $406 \mathrm{~kb}$ )

Additional file 3: Images of brain CT scans of rats under different experimental treatments and densitometric analysis of tissue homogeneity. (PPTX $361 \mathrm{~kb}$ )

\footnotetext{
Abbreviations

AA: Arachidonic Acid; AD: Alzheimer's disease; CNS: Central nervous system; CRP: C-Reactive Protein; CT: Computed tomography; DE: Diabetic encephalopathy; DHA: Docosahexaenoic acid; DM2: Type 2 diabetes mellitus; EPA: Eicosapentaenoic acid; ER: Endoplasmic reticulum; eSS: Stillman-Salgado rats; FA: Fatty acid; FAME: Fatty acids methyl ester; FFA: Free fatty acid; GC: Gas liquid chromatography; GGTP: Gamma glutamyltranspeptidase; HbA1c: Glycated hemoglobin; HE: Hematoxylin-eosin stain; IL: Interleukin; LGCI: Low grade chronic inflammation; NDGA: Nordihydroguaiaretic acid; OGTT: Oral Glucose Tolerance Test; PUFAs: Polyunsaturated fatty acids; SYN: Synaptophysin; TG: Triglycerides
} 


\section{Acknowledgments}

We deeply thanks Dr. L. Poggi for her critical reading and discussion of the MS and to Gina Mazzudulli (CPA-CONICET) for her excellent technical aid.

\section{Funding}

This work was also supported by the funds provided by CONICET, SECYTUNC and SECYT-UNLAR (Argentina).

\section{Availability of data and materials}

The datasets supporting the conclusions of this article are available in the Facultad de Ciencias Médicas of Universidad Nacional de Córdoba repository, http://biologiacelular.webs.fcm.unc.edu.ar/database-diabetic-encephalopathyn3-pufas-and-ndga-in-ess-rats/.

\section{Authors' contributions}

GTD-G, GR, MEP performed the tests, obtained and analyzed the data. CBL and $A D$ collaborate with handling of animals, experiments, data analysis and interpretation. ARE and GR made the experimental design and direction of the study and wrote this work. All authors worked in correction of this manuscript. All authors read and approved the final manuscript.

\section{Ethics approval and consent to participate}

All animal procedures were approved by the local animal care committee (CICUAL, Facultad de Ciencias Médicas, Universidad Nacional de Córdoba, Argentina) and were developed according to international regulations.

\section{Consent for publication}

All authors have read and approved the final version of manuscript for publication.

\section{Competing interests}

The authors declare that they have no competing interests.

\section{Publisher's Note}

Springer Nature remains neutral with regard to jurisdictional claims in published maps and institutional affiliations.

\section{Author details}

'Biología Celular, Histología y Embriología. Facultad de Ciencias Médicas, INICSA CONICET-Universidad Nacional de Córdoba, Córdoba, Argentina. ${ }^{2}$ Geriatric Center "San Ricardo Pampuri", Villa Carlos Paz and Gerontology Committee, Argentine Society of Diabetes, Córdoba, Argentina. ${ }^{3}$ Cátedra de Histología y Embriología, Universidad Nacional de La Rioja (UNLaR), La Rioja, Argentina.

Received: 6 November 2018 Accepted: 30 November 2018 Published online: 08 February 2019

\section{References}

1. Nowlin SY, Hammer MJ, D'Eramo MG. Diet, inflammation, and glycemic control in type 2 diabetes: an integrative review of the literature. J Nutr Metab. 2012;2012:542698.

2. Giovannelli J, Trouiller P, Hulo S, Chérot-Kornobis N, Ciuchete A, Edmé JL, Matran R, Amouyel P, Meirhaeghe A, Dauchet L. Low-grade systemic inflammation: a partial mediator of the relationship between diabetes and lung function. Ann Epidemiol. 2018;28(1):26-32. https://doi.org/10.1016/j. annepidem.2017.11.004.

3. Díaz-Gerevini GT, Repossi G, Dain A, Tarres MC, Das UN, Eynard AR. Cognitive and motor perturbations in elderly with longstanding diabetes mellitus. Nutrition. 2014;30:628-35.

4. Poitout V, Amyot J, Semache M, Zarrouki B, Hagman D, Fontés G Glucolipotoxicity of the pancreatic Beta cell. Biochim Biophys Acta. 2010; 1801:289-98.

5. Hansen JB, Dos Santos LRB, Liu Y, Prentice KJ, Teudt F, Tonnesen M, Jonas JC, Wheeler MB, Mandrup-Poulsen T. Glucolipotoxic conditions induce $\beta$-cell iron import, cytosolic ROS formation and apoptosis. J Mol Endocrinol. 2018; 61(2):69-77. https://doi.org/10.1530/JME-17-0262.

6. Park HR, Kim JY, Park KY, Lee J. Lipotoxicity of palmitic acid on neural progenitor cells and hippocampal neurogenesis. Toxicol Res. 2011;27:103-10.

7. Perez-Martinez P, Perez-Jimenez F, Lopez-Miranda J. n-3 PUFA and lipotoxicity. Biochim Biophys Acta. 2010;1801:362-6.
8. Jafari T, Fallah AA, Azadbakht L. Role of dietary n-3 polyunsaturated fatty acids in type 2 diabetes: a review of epidemiological and clinical studies. Maturitas. 2013;74:303-8.

9. Chan JKW, Bittner S, Bittner A, Atwal S, Shen WJ, Inayathullah M, Rajada J, Nicolls MR, Kraemer FB, Azhar S. Nordihydroguaiaretic acid, a Lignan from Larrea tridentata (creosote bush), protects against American lifestyle-induced obesity Syndrome diet-induced metabolic dysfunction in mice. J Pharmacol Exp Ther. 2018;365(2):281-90. https://doi.org/10.1124/jpet.117.243733.

10. Daniele SM, Montenegro SM, Tarres MC, Picena JC, Martinez SM. The eSS rat, a non-obese model of disordered glucose and lipid metabolism and fatty liver. Diabetol Metab Syndr. 2010;17:2-15.

11. Tarres MC, Martinez SM, Montenegro S, Llorens A, Picena JC, Naves A. The eSS rat. A model of non-insulin-dependent human diabetes. Amer. J Pathol. 1992;141:761-3.

12. Repossi G, Pasqualini ME, Das UN, Eynard AR. Polyunsaturated fatty acids modulate differentially cell proliferation and endocannabinoid system in two human cancer lines. Arch Med Res. 2017;48(1):46-54

13. Gundala NKV, Naidu VGM, Das UN. Arachidonic acid and lipoxinA4 attenuate streptozotocin-induced cytotoxicity to RIN5 F cells in vitro and type 1 and type 2 diabetes mellitus in vivo. Nutrition. 2017;35:65-80. https:// doi.org/10.1016/j.nut.2016.10.004.

14. Greenberg R. The role of neophobia and neophilia in the development of innovative behavior of birds. In: Reader SM, Laland KN, editors. Animal Innovation. Cambridge: University Press; 2003. p. 175-96.

15. Labots M, Van Lith HA, Ohl F, Arndt SS. The modified hole board measuring behavior, cognition and social interaction in mice and rats. J Vis Exp 2015; (98): 52529. doi: https://doi.org/10.3791/52529.

16. Pierard C, Dorey R, Henkous N, Mons N, Béracochéa D. Different implications of the dorsal and ventral hippocampus on contextual memory retrieval after stress. Hippocampus. 2017 Sep;27(9):999-1015. https://doi.org/ 10.1002/hipo.22748.

17. Urakawa S, Takamoto K, Hori E, Sakai N, Ono T, Nishijo H. Rearing in enriched environment increases parvalbumin-positive small neurons in the amygdala and decreases anxiety-like behavior of male rats. BMC Neurosci. 2013;14:13. https://doi.org/10.1186/1471-2202-14-13.

18. Ly H, Verma N, Wu F, Liu M, Saatman KE, Nelson PT, Slevin JT, Goldstein LB, Biessels GJ, Despa F. Brain microvascular injury and white matter disease provoked by diabetes-associated hyperamylinemia. Ann Neurol. 2017;82(2): 208-22. https://doi.org/10.1002/ana.24992.

19. Al Dubayee MS, Alayed H, Almansour R, Alqaoud N, Alnamlah R, Obeid D, Alshahrani A, Zahra MM, Nasr A, Al-Bawab A, Aljada A. Differential expression of human peripheral mononuclear cells phenotype markers in type 2 diabetic patients and type 2 diabetic patients on metformin. Front Endocrinol. 2018;9:537. https://doi.org/10.3389/fendo.2018.00537.

20. Liu Y, Li M, Zhang Z, Ye Y, Zhou J. Role of microglia-neuron interactions in diabetic encephalopathy. Ageing Res Rev. 2018;42:28-39.

21. Toth C, Schmidt AM, Tuor UI, Francis G, Foniok T, et al. Diabetes, leukoencephalopathy and rage. Neurobiol Dis. 2006;23:445-61.

22. van Harten B, de Leeuw FE, Weinstein HC, Scheltens P, Jan Biessels G. Brain imaging in patients with diabetes a systematic review. Diabetes Care. 2006;29:2539-48.

23. Dain A, Repossi G, Díaz-Gerevini GT, Vanamala J, Das UN, Eynard AR. Effect of polyunsaturated fatty acids and nordihydroguaiaretic acid treatment on metabolic and inflammatory markers in a spontaneous type 2 diabetes mellitus model (Stillman Salgado rats) vs control non treated group. Lipids Health Dis. 2016;15(1):205.

24. Nelson PT, Smith CD, Abner EA, Schmitt FA, Scheff SW, et al. Human cerebral neuropathology of type 2 diabetes mellitus. Biochim Biophys Acta. 2009;1792:454-69.

25. Das UN. Metabolic Syndrome Pathophysiology: The Role of Essential Fatty Acids. Ames: Wiley-Blackwell; 2010.

26. Montanaro MA, Lombardo YB, González MS, Bernasconi AM, Chicco A, et al. Effect of troglitazone on the desaturases in a rat model of insulin-resistance induced by a sucrose-rich diet. Prostaglandins Leukot Essent. Fatty Acids. 2005;72:241-50.

27. Poitout V, Robertson RP. Glucolipotoxicity: fuel excess and $\beta$-cell dysfunction. Endocr Rev. 2008;3:351-66.

28. Liu JJ, Raynal S, Bailbé D, Gausseres B, Carbonne C, et al. Expression of the kynurenine pathway enzymes in the pancreatic islet cells. Activation by cytokines and glucolipotoxicity. Biochim Biophys Acta. 2015;1852:980-91.

29. Wei D, Li J, Shen M, Jia W, Chen N, et al. Cellular production of n-3 PUFAs and reduction of $n-6-$ to- $n-3$ ratios in the pancreatic $\beta$-cells and islets 
enhance insulin secretion and confer protection against cytokine-induced cell death. Diabetes. 2010;2:471-8.

30. Maedler K, Spinas GA, Dyntar D, Moritz W, Kaiser N, Donath MY. Distinct effects of saturated and monounsaturated fatty acids on $\beta$-cell turnover and function. Diabetes. 2001;50:69-76.

31. lizuka Y, Kim H, Izawa T, Sakurai K, Hirako S, et al. Protective effects of fish oil and pioglitazone on pancreatic tissue in obese KK mice with type 2 diabetes. Prostaglandins Leukot Essent Fat Acids. 2016;115:53-9.

32. Bast T. Toward an integrative perspective on hippocampal function: from the rapid encoding of experience to adaptive behavior. Rev Neurosci. 2007;18:253-81.

33. Corella D, Asensio EM, Coltell O, Sorlí JV, Estruch R, et al. CLOCK gene variation is associated with incidence of type-2 diabetes and cardiovascular diseases in type-2 diabetic subjects: dietary modulation in the PREDIMED randomized trial. Cardiovasc Diabetol. 2016;7(15):4.

34. Zhao Y, Yan Y, Zhao Z, Li S, Yin J. The dynamic changes of endoplasmic reticulum stress pathway markers GRP78 and CHOP in the hippocampus of diabetic mice. Brain Res Bull. 2014;18:923000195-6.

35. Wayhs $C A$, Mescka CP, Guerreiro G, Moraes TB, Jacques $C E$, et al. Diabetic encephalopathy-related depression: experimental evidence that insulin and clonazepam restore antioxidant status in rat brain. Cell Biochem Funct. 2014:32:711-9

36. Amin SN, Younan SM, Youssef MF, Rashed LA, Mohamady I. A histological and functional study on hippocampal formation of normal and diabetic rats. F1000Res. 2013;9(2):151.

37. Lee SH, Sharma M, Südhof TC, Shen J. Synaptic function of nicastrin in hippocampal neurons. Proc Natl Acad Sci U S A. 2014;17:8973-8.

38. Smith TD, Adams MM, Gallagher M, Morrison JH, Rapp R. Circuit-specific alterations in hippocampal synaptophysin immunoreactivity predict spatial learning impairment in aged rats. Neuroscience. 2000;12:6587-93.

39. Tian X, Liu Y, Ren G, Yin L, An R. Resveratrol limits diabetes-associated cognitive decline in rats by preventing oxidative stress and inflammation and modulating hippocampal structural synaptic plasticity. Brain Res. 2016;1650(1):1-9.

40. Wang JQ, Yin J, Song YF, Zhang L, Ren YX, et al. Brain aging and AD-like pathology in Streptozotocin-induced diabetic rats. J Diabetes Res. 2014;2014:796840.

41. Osimo EF, Cardinal RN, Jones PB, Khandaker GM. Prevalence and correlates of low-grade systemic inflammation in adult psychiatric inpatients: An electronic health record-based study. Psychoneuroendocrinology. 2018;91:226-34.

42. Forbes JM, Cooper ME. Mechanisms of diabetic complications. Physiol Rev. 2013:93:137-88.

43. Pereira CS, Molz P, Palazzo RP, de Freitas TA, Maluf SW, et al. DNA damage and cytotoxicity in adult subjects with prediabetes. Mutat Res. 2013;15:76-81.

44. de la Monte SM, Tong M. Brain metabolic dysfunction at the Core of Alzheimer's disease. Biochem Pharmacol. 2014;88(4):548-59.

45. Devi TS, Hosoya K, Terasaki T, Singh LP. Critical role of TXNIP in oxidative stress, DNA damage and retinal pericyte apoptosis under high glucose: implications for diabetic retinopathy. Exp Cell Res. 2013;15:1001-12.

46. Krause DL, Müller N. Neuroinflammation, microglia and implications for antiinflammatory treatment in Alzheimer's disease. Int J Alzheimers Dis. 2010; 2010:732806.

47. Mülner E, Brath H, Pleifer S, Schiermayr C, Baierl A, et al. Vegetables and PUFA-rich plant oil reduce DNA strand breaks in individuals with type 2 diabetes. Mol Nutr Food Res. 2013;57:328-38.

48. Kapoor R, Kakkar P. Protective role of morin, a flavonoid, against high glucose induced oxidative stress mediated apoptosis in primary rat hepatocytes. PLoS One. 2012;7:e41663.

49. Hajjar T, Meng GY, Rajion MA, Vidyadaran S, Othman F, et al. Omega 3 polyunsaturated fatty acid improves spatial learning and hippocampal peroxisome-proliferator activated receptors (PPARa and PPARY) gene expression in rats. BMC Neurosci. 2012;18(13):109.

50. Dheen ST, Kaur C, Ling EA. Microglial activation and its implications in the brain diseases. Curr Med Chem. 2007;14:1189-97.

51. Feng YM, Zhao D, Zhang N, Yu CG, Zhang Q, et al. Insulin resistance in relation to lipids and inflammation in Type-2 diabetic patients and nondiabetic people. PLoS One. 2016;11:e0153171.

52. Sun $L$, Hou XH, Xue SH, Yan F, Dai YJ, et al. Fish oil modulates glycogen synthase kinase-3 signaling pathway in diabetes-induced hippocampal neurons apoptosis. Brain Res. 2014;29:37-49.

53. Dobrian AD, Morris MA, Taylor-Fishwick DA, Holman TR, Imai Y, Mirmira RG, Nadler JL. Role of the 12-lipoxygenase pathway in diabetes pathogenesis and complications. Pharmacol Ther. 2018. https://doi.org/10.1016/j. pharmthera.2018.10.010.53.

Ready to submit your research? Choose BMC and benefit from:

- fast, convenient online submission

- thorough peer review by experienced researchers in your field

- rapid publication on acceptance

- support for research data, including large and complex data types

- gold Open Access which fosters wider collaboration and increased citations

- maximum visibility for your research: over $100 \mathrm{M}$ website views per year

At BMC, research is always in progress.

Learn more biomedcentral.com/submissions 